\title{
Organ-preserving technologies and their effectiveness in patients with placenta accreta
}

\begin{abstract}
Summary
In recent years, in practical obstetrics, placental adherence into the myometrium, diagnosed after the birth of the fetus during caesarean section or in the 3rd period of labor, has become increasingly common. It causes massive bleeding, hysterectomy and maternal mortality.
\end{abstract}

Purpose: To assess the outcome of childbirth with organ-preserving technology in cases of placenta accreta.

Material and methods: 56 women in placenta accreta were observed and delivered for 2016-2018. 49 women delivered by Cesarean section and 7 women gave vaginal delivery. Two methods were used to preserve the uterus during placenta accreta: the first method -35 women who have laparotomy, a cesarean section with a section on the uterus above the scar and above the edge of the placenta, ligation of the uterine arteries, excision of the incremental area and metroplasty, imposition of compression sutures on the uterus. The second method -7 women after vaginal delivery left the whole or part of the placenta in the uterus - placenta in situ. The risk of placenta accreta was placenta previa, scar on the uterus, abortions in anamnesis, over 30 years of age third or more births.

Results and discussion: From 35 pregnant women who underwent an organ-preserving procedure for caesarean section, $33(94,3 \%)$ women managed to preserve the uterus, the remaining $2(5,7 \%)$ women underwent hysterectomy of 7 cases of placenta in situ, in 2 $(28,6 \%)$ cases a hysterectomy was performed и. and uterus was preserved in $5(71.4 \%)$ women.

Conclusions: The effectiveness of improved organ-preserving technology to preserve the reproductive function of women with placenta accreta is high. In the group with the organpreserving method with placenta, the volume of blood loss, the volume of transfusion of blood components is much lower than in the hysterectomy group.

Keywords: placenta accreta, organ-preserving technique, cesarean section, placenta in situ, obstetric hemorrhage
Volume II Issue 5 - 2020

\author{
Babazhanova Sh D, Lyubchich AS, Djabbarova \\ Yu K \\ Obstetrician-gynecologist, Republican Perinatal center of the \\ Ministry of Health care Tashkent, Uzbekistan
}

\begin{abstract}
Correspondence: Prof. Djabbarova Yulduz Kasimovna, DSc Obstetrician-gynecologist, Republican perinatal center of the Ministry of Health, Dj.Abidova St. 223, Tashkent, 100140 Republic of Uzbekistan, Tel +998946996402, Email ulduzjab3@mail.ru
\end{abstract}

Received: August 12,2020 | Published: September 07, 2020

\section{Introduction}

Obstetric bleeding is the leading maternal mortality pattern worldwide, especially in developing countries..$^{1-5}$ Recently, bleeding caused by the implantation of the placenta into the walls of the uterus has become increasingly among the causes. Many authors attribute this to an increase in the frequency of abdominal delivery. ${ }^{6,7}$ In the USA, where the frequency of caesarean section is up to $33 \%$, hysterectomy due to the placenta accreta was carried out in $37 \% .{ }^{8}$ In our country, according to national standards for placental accreta, hysterectomy is also recommended. ${ }^{9}$ The increased incidence of massive bleeding in the placenta accreta, which is accompanied by a loss of reproductive function after childbirth, dictates the need to find alternative ways to solve this problem. In this regard, in recent years, some researchers ${ }^{10}$ proposed organ-preserving methods in placenta accreta.

JM Palacios-Jaraquemada et al. ${ }^{10}$ for the first time published a method of preserving the uterus, consisting in excising an overstretched and incapacitated section of myometry together with its underlying placenta. ${ }^{10} \mathrm{E}$ Chandraharan et al. ${ }^{11}$ and MA Kurzer ${ }^{12}$ improved the method JM Palacios Jaraquemada, supplementing it with balloon occlusion of the internal iliac arteries or common iliac arteries. The frequency of detection of the placenta accreta in pregnant women in the Republican Perinatal Center of Uzbekistan, which is a level 3 institution, and where pregnant women with severe somatic or obstetric pathology are concentrated, has increased in recent years, which prompted us to looking for a new solutions to preserve reproductive function in women.

Purpose: To assess the outcome of childbirth with organ-preserving technology in cases of placenta accreta.

\section{Material and research methods}

In the Republican Perinatal Center of Uzbekistan, 56 pregnant women observed placenta accreta for 2016-2018. A history was collected in all pregnant women, general clinical and obstetric studies were carried out, ultrasound and Doppler vascular examination of the uterus and fetus were determined. Ultrasound studies (2D) with color Doppler display (Voluson E8, expert GE Healthcare) were carried out. Ultrasound was the main method of prenatal diagnosis of placenta accreta and was performed transabdominally and transvaginally. The diagnosis of placenta accreta was made by combining several ultrasound signs: the disappearance of the hypoechoic retroplacental zone; thinning subplacental myometry; multiple placental vascular lacunae; increased vascularization of lower uterine segment 
myometry; there is no boundary between the uterine wall and the bladder. To identify risk factors for placenta accreta, data from 56 women with placenta accreta and 5361 women without placental abnormalities were studied.

Depending on the method of delivery, women with placenta accreta were divided into 3 groups: a modified single-step organ-preserving surgical method for placenta accreta was used in 35 women with caesarean section (main group 1), the comparison group (group 2) was 14 women with placenta accreta, who underwent hysterectomy without trying to preserve the organ. Group 3 was made up of 7 women with placenta accreta in independent childbirth.

We used the following 2 methods to preserve the uterus in the placenta accreta: the first method was used (35 patients) in caesarean section - laparotomy, caesarean section, vascular ligation, excision of the incremental area and metroplasty, application of compression sutures on the uterus. The second method was used in vaginal childbirth (7 patients): leaving all or part of the placenta in the uterus - placentas in situ. The volume of blood loss during and after surgery, the level of hemoglobin before and after surgery, the volume of blood transfusion, the duration of patients in the intensive care unit, and the postpartum department was studied.

\section{Results and discussion}

It was found that factors of high risk of placenta accreta in Uzbekistan include: age of pregnant women over 30 years $(\mathrm{OR}=2.0)$, third $(\mathrm{OR}=2.0)$, fifth $(\mathrm{OR}=5.9)$ births, history of abortion $(\mathrm{OR}=13.9)$. Among the risk factors for placenta accreta, the highest risk had the fact of placenta previa (OR 381.362, 95\% CI 7.994-24.063), the next high factor is the scar on the uterus, which was 17 times higher than the control group (OR 17.016, CI 9.572-30.249) at the same time, twice the scar on the uterus increased the risk by 8 times (OR 8.562 , CI 4.605-15.922), triplicate - 9 times (OR 9.443. 2.166-41.157).

The gestational age of labor is $28-33$ weeks - in $12(26.7 \%), 34-36$ weeks - in $9(20.0 \%)$ and 37 weeks or more - in 24 (53.3\%) women. Placenta previa was diagnosed in $24(53.3 \%)$, arterial hypertension in $11(24 \%)$, IDA- in $9(20 \%)$, escalation of chronic pyelonephritis in $4(9 \%)$, fetal abnormality in $6(13 \%)$, uterine myoma in $2(4.5 \%)$, infertility + IVF - in 2 (4.5\%). In 1group, a Joel Cohen laparotomy was performed in 9 patients, and 26 patients in lower-middle access. In our experience, it is rational for pregnant women diagnosed with placenta accreta to perform laparotomy with a lower medial incision, since the risk of bleeding is less, as well as more access to the small pelvis and uterus, to retroperitoneal space, which creates convenience if the volume of surgery needs to be further expanded to hysterectomy or ligation of the internal iliac arteries.

We applied an improved organ-preserving technique in placental growth. Our method of preserving the uterus with placenta accreta differs from other methods in that during caesarean section in women with a uterine scar diagnosed with "growth in the scar area" we did not perform a bottom caesarean section, as some authors recommend, ${ }^{6,12}$ in which two incisions are made on the uterus - on the bottom of the uterus to extract the fetus and on the lower segment of the uterus in the area of the scar for metroplasty, which leads to the formation of two scarring on the uterus on the bottom of the uterus and on the lower segment, and increases the risk of rupture of the uterus during subsequent pregnancy. An incision in the uterus we made transverse above the edge of the placenta and the edge of the scar on the uterus so as not to cut the placenta, which can cause abundant bleeding. The incision was made by an acute method, according to the Derfler method, and, after removing the child, we did not separate the placenta from the uterus, applied ligatures to the uterine arteries, round ligaments and utero-ovarian arteries right under the junction with the uterus of our own ovarian ligament. Preliminary ligation of the vessels of the uterus led to a decrease in blood loss. After that, the placenta was removed, and if the placenta was in the area of the thin scar, the scar along with the placenta was cut out and metroplasty was carried out. On the placenta bed, if it was located in the area of the inner palate or posterior wall, hemostatic "P" or "Z" sutures were placed inside with chrome cetgut or vicryl suture on the uterine mucosa, and, depending on the place of bleeding, several compression sutures were applied to the uterus with the sides of the serous membrane along Cho, Pereira or "P" or "Z". In case of continuing bleeding, the internal iliac arteries were ligated. At the same time, measures were taken to replenish the volume of circulating blood with crystalloids, blood preparations and intravenous administration of uterotonics, tranexamic acid and antibiotics. The volume of blood loss was carefully controlled, in case of severe bleeding or blood loss exceeding $2500 \mathrm{ml}$, further uterine preservation tactics were stopped and hysterectomy was performed. $1^{\text {st }}$ groups and $2^{\text {nd }}$ were not statistically different between each other by age, parity, clinical and anamnestic characteristics, which makes these groups statistically homogeneous and makes it possible to compare the intervention. The average age of women with ingrowth was $30.7 \pm 5$ years. $37.1 \%$ had a history of abortions, the scar on the uterus after the previous CS was in $69 \%$, while 1 scar - in $41 \%, 2$ scars $-25 \%, 3$ or more scars - in $3.4 \%$ of women, placenta previa was diagnosed in $67.8 \%$, arterial hypertension in $14.3 \%$, obesity in $10.8 \%$ of women, anemia was detected in one in three $53.1 \%$ of women had a premature operative labor, while 46.9 per cent of pregnant women had a full-time labor.

In the main group, 26 women had inferior LT, 9 had Joel Coen LT in view of the old postoperative scar, 8 women had median LT in the control group, 6 had Joel Coen LT.

Lower-medial access is more preferable, since during surgery, indications are possible for additional procedures and expansion of the operation volume, for example, ligation of the iliac arteries, dissection of adhesions, hysterectomy, etc. The duration of the operation in $1^{\text {st }}$ group is ranged from 55 minutes to 128 minutes, in $2^{\text {nd }}$ group is ranged from 105 minutes to 250 minutes. The infusion program and uterotonics were administered according to the caesarean section standard and the postpartum bleeding standard. After the first dose of antibiotics, after 1 hour, a prophylactic dose of antibiotics was re-administered. In group No. 1 in 26 cases there was placenta, in group No. 2 - in 12 cases. In the main group No. 1 in 28 (80\%) cases, and in the control group in $12(85.7 \%)$ there was mainly the front location of the placenta (front wall + inner reed, front wall+side wall, front wall+inner reed+rear wall), in the remaining $7(20 \%)$ cases in the main and $2(14.3 \%)$ in the control group - the rear side wall and rear wall + front wall. In addition to ligation of uterine vessels, round ligaments and ovarian-uterine arteries, in 3 cases internal iliac arteries were ligated due to bleeding. In the control group, in 4 (28.5\%) cases, the average blood loss in the main group was $1250 \pm 158 \mathrm{ml}$, in the control - $2340 \pm 172 \mathrm{ml},(\mathrm{p}<0.01)$. Hemotransfusion in the main group was carried out $82.9 \%$, in the control group-completely sick, but the data did not differ statistically. In the uterine extirpation group, er. weight from 430 to $2120 \mathrm{ml}$, median was $720 \mathrm{ml}$, in the main group - transfused from 220 to $1130 \mathrm{ml}$, median was $355 \mathrm{ml}$ of red blood cells $(\mathrm{p}<0.01)$. STP Freshly frozen plasma (FFP) transfusion was performed in the main group in $82 \%$, and in the uterine extirpation group in $44 \%$ of patients $(\mathrm{p}<0.05 \%)$. 
Of the 35 cases of placenta accreta, $33(94.2 \%, 33 / 35)$ of women in the first group who underwent the above method of cesarean section with metroplasty. in cases, successful organ-preserving tactics were performed and the uterus. In 2 cases $(5.8 \%)$ the uterus could not be preserved and hysterectomy was carried out due to massive bleeding. The average length of stay in the hospital in the main group was $9.6 \pm 2.3$ days, in the control group - $11 \pm 3.1$ days the maternity hospitals were in the hospital not only for complications of the postpartum period, but in connection with preterm birth, for the care of the child.

Group 3 consisted of 7 women giving birth vaginally. In the postpartum period, all patients had early postpartum bleeding, conservative measures were taken to stop bleeding, a manual examination of the uterine cavity, during which the placenta accreta was diagnosed. Of the 7 patients in $2(28.6 \%)$ cases, hysterectomy was performed due to massive postpartum bleeding. In 5 (71.4\%): in cases, an organ-preserving technique was carried out - the placenta in situ, a whole or part of the placenta was left in the uterus. All these patients performed 1 course of methotrexate in the postpartum period. In 2 women, pieces of the placenta departed on their own. The remaining 3 women underwent uterine curettage, the remaining parts of the placenta were removed, two women within 12-14 days, and one placenta was removed 43 days after delivery. Patients in whom, after vaginal delivery, the entire placenta remains in the uterus deserve attention. This patient $(2.2 \%)$ developed metroendometritis within 23 days; she underwent comprehensive treatment of metroendometritis. Received 3 courses of metrotrexate. On day 43, she was readmitted to the hospital, where after training, the placenta was completely removed from the uterus.

\section{Conclusion}

The effectiveness of improved organ-preserving technology to preserve the reproductive function of women with placenta accreta is high. In the group with the organ-preserving method with placenta, the volume of blood loss, the volume of transfusion of blood components is much lower than in the hysterectomy group. Thus, the use of an improved organ-preserving technique in the placenta accreta during caesarean section leads to the preservation of the reproductive function of women in $94.2 \%$ and in vaginal childbirth in $71.4 \%$ of cases.

\section{Acknowledgments}

None.

\section{Funding}

The authors of this article confirm the lack of financial or any other support that needs to be reported.

\section{Conflicts of interest}

The authors confirm the absence of any other conflict of interest that needs to be reported.

\section{References}

1. Carroll M, Daly D, Begley CM. The prevalence of women's emotional and physical health problems following a postpartum haemorrhage: a systematic review. BMC Pregnancy Childbirth. 2016;16:261.

2. Eimer G, O'Milley, Popivanov P. Material near miss: What lies beneath? Europ Obstet Gynecol Reprod Bidol. 2016;199:116-119.

3. Le Gouez A, Mercier FJ. Major obstetric hemorrhage. Transfus Clin Biol. 2016;23(4):229-232.

4. Strategies toward end ingpreventablem at ernal mortality (EPMM) Geneva: World Health Organization; 2015.

5. Volkov VG, Granatovich NN, Guseva KM. Obstetric bleeding in the structure of the causes of MS in the Tula region. Akusherstvo Ginekologiya i Reproduktologiya. 2017;11(1):31-35.

6. Latyshkevich OA. Placenta accreta in patients with a scar on the uterus after cesarean section. Organ-preserving operations: dis. can. honey. Sciences; 2015. 133 p.

7. Vukolova VA. Proofilactics of hypotonic bleeding during repeated caesarean section in pregnant women with low placenta location: Autoref dissedging honey. 2018.24 p.

8. Hernandez JS, Nuangchamnong N, Ziadie M, et al. Placental and uterine pathology in women undergoing peripartum hysterectomy. Obstet Gynecol. 2012;119(6):1137-1142.

9. National standards for obstetrics. Tashkent; $2015.64 \mathrm{p}$.

10. Palacios-Jaraquemada JM, Pesaresi M, Nassif JC, et al. Anterior placenta percreta: surgical approach, hemostasis and uterine repair. Acta Obstet Gynecol Scand. 2004;83(8):738-744.

11. Chandraharan E, Rao S, Belli AM, et al. The triple-P procedure as a conservative surgical alternative to peripartum hysterectomy for placenta percreta. Int J Gynecol Obstet. 2012;117:191-194.

12. Kurtser MA, Yu I, Breslav AM, et al. Experience in the use of temporary balloon occlusion of the common iliac arteries during organ-preserving operations in patients with an increase in the placenta. Akusherstvo ginekologiya. 2013;7:80-84. 